Im Allgemeinen wird nun auch $\gamma_{1}$ als unendlich gross anzunehmen sein, weil gleiches von den Zahlen $s, z_{1}-z_{0}$, $z_{1}-v$ und $z_{1}$ gilt, während $\frac{z_{1}-v}{z_{1}}$ ein endlicher Bruch bleibt. Unter dieser Voraussetzung ist einfach:

$$
W_{\gamma}=\frac{2}{\sqrt{\pi}} \int_{0}^{\gamma / \sqrt{-1 / 2 z_{1} \sigma^{\prime}}} e^{-x^{2}} \mathrm{~d} x
$$

Das wahrscheinlichste Resultat ist also $z=z_{1}$, wo $z_{1}$ durch (1 2) gegeben ist und der wahrscheinliche Fehler $r$ dieses Resultates ist:

2 r)

$$
r= \pm 0.67449 \sqrt{z_{1}} \cdot \sqrt{\frac{z_{1}\left(z_{1}-\nu\right)}{s \cdot\left(z_{1}-z_{0}\right)}}
$$

Als Prüfung der letzten beiden Formeln kann $n_{3}=0$, $c_{3}=c^{\prime}, c_{2}=c_{1}=0$ gesetzt werden; dann erhält man:

$$
\begin{gathered}
z_{1}=\frac{n_{1} n_{2}}{c^{\prime}} \\
r= \pm 0.67449 \sqrt{\frac{n_{1} n_{2}\left(n_{1}-c^{\prime}\right)\left(n_{2}-c^{\prime}\right)}{c^{\prime} c^{\prime} c^{\prime}}}
\end{gathered}
$$

und dies stimmt, wie es sein muss mit Formel (6), wenn die passende Buchstabenveränderung vorgenommen wird.

Als specieller Fall sei weiter hervorgehoben:

Dann wird

$$
n_{1}=n_{2}=n_{3}=n
$$

$$
v=3^{n-s, s}=2 c+c_{1}+c_{2}+c_{3}
$$

und die Formel (I 2 ) giebt:

$$
\begin{aligned}
& z_{1}=\frac{3 n^{2}}{2 s}\left(\mathrm{I}+\sqrt{\mathrm{I}-\frac{4 s}{9 n}}\right) \\
& z_{0}=\frac{3 n^{2}}{2 s}\left(\mathrm{I}-\sqrt{\mathrm{I}-\frac{4 s}{9 n}}\right)
\end{aligned}
$$

nach (I 7 ) findet man:

$$
\sigma^{\prime}=-\frac{3 n^{2} \sqrt{1-\frac{4 s}{9 n}}}{z_{1}{ }^{2}\left(\varepsilon_{1}-v\right)}
$$

Setzt man z. B.:

$$
\begin{aligned}
& n=3^{60} \\
& s=17^{\circ}
\end{aligned}
$$

so wird:

$$
\begin{aligned}
& z_{1}=2160, z_{0}=\frac{2160}{17} \\
& \sigma^{\prime}=-\frac{2}{3375^{\circ}}
\end{aligned}
$$

und also:

$$
r= \pm 0.67449 \sqrt{\frac{33750}{2}}= \pm 87.6
$$

Das wahrscheinlichste Resultat ist hier also:

$$
2160 \pm 88 .
$$

Wendet man die Formel (19) an, so findet sich:

$$
\gamma_{1}=6.8 \mathrm{I}
$$

und obwohl dies ein ziemlich kleiner Werth ist, so wird doch schon:

$$
\frac{2}{\sqrt{\pi}} \int_{0}^{\gamma_{1}} e^{-t^{2}} \mathrm{~d} t .
$$

bis auf ungefähr zwanzig Ziffern der Einheit gleich und infolge dessen der Werth des w. F. $r$ gar nicht mehr geändert.

München 1882 im December.

\section{$H$. Seeliger.}

\title{
Neue Methode zur Berechnung von Doppelsternbahnen.
}

Obgleich es schon eine Menge von Methoden giebt, die Bahn eines Doppelsternes zu finden, wird es kaum nöthig sein, sich zu entschuldigen, wenn man die Anzahl noch vermehrt. Es giebt eine solche Mannigfaltigkeit von Formen der Bahnen und Eigenthümlichkeiten der Beobachtungen, dass die Hülfsmittel auch viele Verschiedenheit darbieten müssen, wenn es immer gelingen soll, jede Aufgabe zu lösen. Eine kleine $Z$ ahl von fein ausgearbeiteten Methoden, wie man solche für Cometen und Planeten hat, wird hier nicht ausreichen. Jede Methode kann bei Doppelsternen nur anzeigen, wie die grösseren Schwierigkeiten besiegt werden können. Der Rechner muss zwischen der 
möglichst grossen Zahl von Methoden wählen können, und auch fähig sein, die gewählte Methode nach den Umständen zu modificiren.

Die hier darzustellende Methode ist zwar ziemlich allgemein anwendbar, zeichnet sich aber besonders dadurch aus, dass sie sich auch in dem Falle anwenden lässt, wenn die Bahnebene die Gesichtslinie enthält (Neigung $=90^{\circ}$ ), für welche es, wenn ich nicht irre, bisher keine Methode gab. -

Ich gehe von folgenden Formeln der scheinbaren Bewegung aus:

$$
t=T+\frac{I}{\mu}(E-e \sin E)
$$

(2) $x=r \cos R=a \cos A(\cos E-\ell)+b \cos B \sin E$

(3) $y=r \sin R=a \sin A(\cos E-c)+b \sin B \sin E$ wo $\boldsymbol{x}$ und $\boldsymbol{y}$ die der Zeit $\boldsymbol{t}$ entsprechenden, rechtwinkeligen
Coordinaten (senkrecht zur Gesichtslinie) sind, also $r=$ Distanz, $R=$ Positionswinkel, $E$ ist die entsprechende excentrische Anomalie.

Die Elemente sind:

$$
\begin{aligned}
T= & \text { Perihelzeit, } \\
\mu= & \text { mittlere jährliche Bewegung, } \\
e= & \text { Excentricität, } \\
a= & \text { Länge und } A=\text { Positionswinkel des Halb- } \\
& \text { messers der scheinbaren Bahn, der durch } \\
& \text { den Hauptstern geht, für welchen } E=0^{\circ}, \\
b= & \text { Länge und } B=\text { Positionswinkel des con- } \\
& \text { jugirten Halbmessers, für welchen } E=90^{\circ} .
\end{aligned}
$$

Die Wahl der letzten 4 der Elemente ist durch einige Vorzüge motivirt gegenüber den gewöhnlichen s wahren Elementen. Unsere Elemente lassen sich unzweideutig bestimmen und geben die leichteste Rechnung der Ephemeride nach:

$$
\begin{aligned}
& r \cos \left[R-\frac{\mathrm{I}}{2}(A+B)\right]=(b \sin E+a \cos E-a e) \cos \frac{\mathrm{I}}{2}(B-A) \\
& r \sin \left[R-\frac{\mathrm{I}}{2}(A+B)\right]=(b \sin E-a \cos E+a e) \sin \frac{\mathrm{I}}{2}(B-A)
\end{aligned}
$$

ausserdem lässt sich nach ihnen die scheinbare Bahn sehr bequem zeichnen. Von den sonst gebräuchlichen wahren Elementen hat nur der mittlere Abstand $\alpha$ einiges Interesse, der Knoten $\Omega$, die Neigung $I$ und der Winkel $\boldsymbol{A}$ zwischen Perihel und Knoten beziehen sich für jeden Stern auf eine besondere Fundamentalebene. Für die Reduction dieser. Elemente auf die unsrigen hat man:

$$
\text { (4) }\left\{\begin{aligned}
\alpha & =\gamma \cos \Gamma \\
\alpha \cos I & =\gamma \sin \Gamma
\end{aligned}\right.
$$

$$
\left\{\begin{array}{l}
\cos G \cos (A-\Omega)=\cos \Gamma \cos A \\
\cos G \sin (A-\Omega)=\sin \Gamma \sin A \\
\sin G \cos (B-\Omega)=-\cos \Gamma \sin A \\
\sin G \sin (B-\Omega)=\sin \Gamma \cos A
\end{array}\right.
$$

$$
\left\{\begin{array}{l}
a=\gamma \cos G \\
b=\sqrt{1-e^{2}} \gamma \sin G
\end{array}\right.
$$

Für die entgegengesetzte Rechnung hat man zuerst aus (6) $\gamma$ und $G$ zu bestimmen, dann statt (5) $\Gamma, A$ und $\Omega$ aus folgenden Glejchungen so $z \mathrm{u}$ bestimmen:

$$
\begin{aligned}
\cos 2 \Gamma \cos 2 A & =\cos 2 G \\
\cos 2 \Gamma \sin 2 A & =-\sin 2 G \cos (B-A) \\
\sin 2 \Gamma & =\sin 2 G \sin (B-A) \\
\cos 2 \Gamma \cos (B+A-2 \delta) & =\cos (B-A) \\
\cos 2 \Gamma \sin (B+A-2 \delta) & =\cos 2 G \sin (B-A),
\end{aligned}
$$

dass $A$ und $A-\Omega$ in denselben Quadranten fallen. Schliesslich $\alpha$ und $I$ aus (4). Integrale

Unsere Methode beruht auf dem Umstande, dass die

$$
\int \frac{\partial t}{x^{2}} \text { und } \int \frac{\partial t}{y^{2}}
$$

sowohl aus den Beobachtungen durch mechanische Quadratur sich berechnen lassen, als auch in recht einfacher Form als Functionen der excentrischen Anomalie und der Elemente dargestellt werden können; es ist nämlich:

$$
\begin{aligned}
& \int \frac{\partial t}{x^{2}}=C-\frac{1}{2 \mu a b \cos A \cos B} \frac{a \cos A(\cos E-e)-b \cos B \sin E}{a \cos A(\cos E-e)+b \cos B \sin E} \\
& \int^{\circ} \frac{\partial t}{y^{2}}=C^{\prime}-\frac{1}{2 \mu a b \sin A \sin B} \frac{a \sin A(\cos E-e)-b \sin B \sin E}{a \sin A(\cos E \quad e)+b \sin B \sin E}
\end{aligned}
$$

Bestimmt man hieraus die bestimmten Integrale für die Zeit zwischen $t_{1}$ und $t_{2}$ und bezeichnet man mit $x_{1}, y_{1}, x_{2}$ und $y_{2}$ die diesen Zeiten entsprechenden Werthe von $x$ und $y$, dann kommt man auf eine sehr merkwürdige Formel: 


$$
\begin{aligned}
x_{1} x_{2} \int_{t_{1}}^{t_{2}} \frac{\partial t}{x^{2}} & =y_{3} y_{2} \int_{t_{1}}^{t_{2}} \frac{\partial t}{y^{2}}=\frac{\mathrm{I}}{\mu}\left[\sin \left(E_{2}-E_{1}\right)-\ell\left(\sin E_{2}-\sin E_{1}\right)\right] \\
& =\frac{\mathrm{I}}{c} r_{1} r_{2} \sin \left(R_{2}-R_{1}\right)=\frac{\mathrm{I}}{c} r_{1} r_{2} \sin \left(c \int_{t_{1}}^{t_{2}} \frac{\partial t}{r^{2}}\right)=\frac{\mathrm{I}}{c}\left(x_{1} y_{2}-x_{2} y_{1}\right)
\end{aligned}
$$

wo $1 / 2 e$ das constante Verhältniss zwischen Areal und Zeit für die scheinbare Bahn ist. Vermittelst ( 1 ) geht aus (7) noch die Gleichung hervor:

$$
t_{2}-t_{1}-x_{1} x_{2} \int_{t_{1}}^{t_{2}} \frac{\partial t}{x^{2}}=\frac{\mathbf{I}}{\mu}\left[E_{2}-E_{1}-\sin \left(E_{2}-E_{1}\right)\right]
$$

Die linke Seite dieser Gleichung ist bekannt; wäre auch die Umlaufszeit und dadurch $\mu$ gegeben, könnte die Differenz, $E_{2}-E_{1}$, der excentrischen Anomalien direct vermittelst der vorhandenen Tafeln über $x-\sin x$ berechnet werden.

Ist die Umlaufszeit unbekannt, dann werden die drei Gleichungen (8), die man mit den drei Zeiten $t_{1}$, $t_{2}$, $t_{3}$ und den entsprechenden Coordinaten und Integralen bilden kann:

$$
\begin{aligned}
& t_{3}-t_{2}-x_{3} x_{2} \int_{t_{2}}^{t_{3}} \frac{\partial t}{x^{2}}=\frac{\mathrm{I}}{\mu}\left[E_{3}-E_{2}-\sin \left(E_{3}-E_{2}\right)\right] \\
& t_{1}-t_{3}-x_{1} x_{3} \int_{t_{3}}^{t^{\prime}} \frac{\partial t}{x^{2}}=\frac{\mathrm{I}}{\mu}\left[E_{1}-E_{3}-\sin \left(E_{1}-E_{3}\right)\right] \\
& t_{2}-t_{1}-x_{2} x_{1} \int_{t_{1}}^{t_{2}} \frac{\partial t}{x^{2}}=\frac{\mathrm{I}}{\mu}\left[E_{2}-E_{1}-\sin \left(E_{2}-E_{1}\right)\right]
\end{aligned}
$$

auch drei Unbekannte $\mu, E_{2}-E_{1}$ und $E_{3}-E_{2}$ enthalten, die man durch Hypothesen über $\mu$ sehr leicht bestimmen kann. Freilich setzt man dabei 8 unter einander unabhängige Zahlen als gegeben voraus, während nur 7 Elemente zu berechnen sind, man könnte also im Allgemeinen nicht alle die gegebenen Zahlen genau darstellen. Doch ist hier die Bedingungsgleichung, welche zwischen den 8 gegebenen Zahlen besteht, in sehr einfacher Form zugänglich. Aus (7) sieht man, dass für jedes Intervall $\int_{t_{1}}^{t_{2}} \frac{\partial t}{x^{2}}$ der Grösse $\frac{y_{2}}{x_{2}}-\frac{y_{1}}{x_{1}}$ proportional sein soll, oder dass $c \int^{\frac{t}{\partial t}} \frac{t}{x^{2}}+d=\frac{y}{x}=\operatorname{tang} R$, wo $d$ wie $c$ constant ist. Man kann also sehr leicht die bestimmten Integrale mit den Coordinaten ausgleichen. Dreiecksflächen:

Wenn $\mu$ und die Differenzen der excentrischen Anomalien gefunden sind, berechnet man mit den doppelten

$$
\triangle_{n, m}=x_{n} y_{m}-x_{m} y_{n}=r_{n} r_{m} \sin \left(R_{m}-R_{n}\right)=c x_{n} x_{m} \int_{i_{n}}^{{ }^{t} m} \frac{\partial t}{x^{2}}
$$

$e$ und $E_{2}$ aus

$$
\begin{aligned}
e \cos E_{2} & =\frac{\triangle_{23} \sin \left(E_{2}-E_{1}\right)-\triangle_{12} \sin \left(E_{3}-E_{2}\right)}{\triangle \triangle_{23}+\triangle_{31}+\triangle_{12}} \\
e \sin E_{2} & =\frac{\triangle_{23} \cos \left(E_{2}-E_{1}\right)+\triangle_{31}+\triangle_{12} \cos \left(E_{3}-E_{2}\right)}{\triangle \triangle_{23}+\triangle_{31}+\triangle_{12}}
\end{aligned}
$$

so wie $E_{1}=E_{2}-\left(E_{2}-E_{1}\right)$ und $E_{3}=E_{2}+\left(E_{3}-E_{2}\right)$. Zuletzt geben zwei der Gleichungen (2) $a \cos A$ und $b \cos B$, zwei Gleichungen (3) $a \sin A$ und $b \sin B$, und jede Gleichung (I) einen Werth von $T$ mit Prüfung der Rechnung. 
Jede rechtwinklige Coordinate $x$ wie $y$ wird zweimal in jedem Umlaufe $=0$, folglich $\frac{1}{x^{2}}=\infty$, zwar bleibt das Integral $\int \frac{\partial t}{x^{2}}$ dabei endlich, es entsteht jedoch aus diesem Umstande einige Schwierigkeit für die numerische Integration. Gewöhnlich kann man diese Schwierigkeit durch verschiedene Mittel besiegen, von welchen wir das vorzuiglichste angeben wollen.

So wie das Flächengesetz:

$$
x \frac{\partial y}{\partial t}-y \frac{\partial x}{\partial t}=c=r^{2} \frac{\partial R}{\partial t}
$$

in den andern Doppelsternbahnmethoden durch vorausgehende Ausgleichungen der Beobachtungen eine wichtige Rolle spielt, so auch in dieser, denn die verschiedenen
Gleichungen (7) sind eben nur neue Ausdrücke für dieses Gesetz, die man durch directe Integration von (9) nach Division dieser Gleichung mit $x^{2}, y^{2}$ oder $r^{2}$ leicht ableitet. (Es versteht sich, mit Ausnahme des Ausdruckes (7), in welchen die excentrischen Anomalien eingehen.) Die Relationen zwischen den verschiedenen Integralen, die gleichzeitig berechnet werden können, bieten vielfache Gelegenheiten zur Ausgleichung derjenigen Data, die zur Berechnung der Elemente angewendet werden sollen. Diese Controle kann in Allgemeinen nach Belieben vervielfältigt werden, denn wie man sieht, ist der Werth von $x_{1} x_{2} \int_{t_{1}}^{t_{2}} \frac{\partial t}{x^{2}}$ von der Richtung und der Einheit der bezüglichen Coordinatenaxe ganz unabhängig; man hat für beliebige Werthe von $m$ und $n$ :

$$
x_{1} x_{2} \int_{t_{1}}^{t_{2}} \frac{c t}{x^{2}}=\left(m x_{1}+n y_{1}\right)\left(m x_{2}+n y_{2}\right) \int_{t_{1}}^{t_{2}} \frac{\partial t}{(m x+n y)^{2}}
$$

Am besten bestimmt sich das Integral, wenn die Richtung der Axé oder $\frac{m}{n}$ so gewählt wird, dass in der Zwischenzeit $t_{1}$ bis $t_{2}, m x+n y$ nahe einen Maximalwerth hat. Wenn nur die Neigung nicht nahe $90^{\circ}$ ist, werden $x, y$ und $r$ nicht alle gleichzeitig $=0$, durch passende Werthe von $\frac{m}{n}$ wird man daher das Integral auch dann scharf bestimmen können, wenn $x=0$.

Wenn jedoch die Neigung so gross ist, dass der Begleiter in den Strahlen des Hauptsternes verschwindet, wenn $x$ und zugleich auch $y$ und $r$ klein sind, dann wird man $\int \frac{\partial t}{x^{2}}$ für solche Zeiten nicht unmittelbar berechnen können, denn nur selten wird man mittelst einer Interpolationsformel die Beobachtungen vor und nach dem Durchgang so genau darstellen können, dass man mit hinlänglicher Schärfe das Integral aus der Interpolationsformel ableiten könnte. In diesem Falle, also wenn mit unseren Elementen näherungsweise $B=A$ oder $180^{\circ}-A$ wird, wird man sehr häufig entweder zwischen $t_{1}$ und $t_{2}$ oder zwischen $t_{2}$ und $t_{3}$ unser
Integral nicht kennen. Zugleich hört eben in diesem Falle die Bahnbestimmung mittels dreier Zeiten und der entsprechenden Coordinaten und Integrale auf, ein uberbestimmtes Problem zu sein. Im Grenzfalle hat man, abgesehen von dem constanten Positionswinkel, nur darin 5 gegebene Zahlen und 5 Elemente; wir müssen also, wenn uns ein Integral abgeht, noch für eine Zeit $t_{4}$ die Distanz oder die Coordinate zur Berechnung heranziehen. Wenn nun ein ganzer Umlauf beobachtet ist, und viel fruher werden derartige Rechnungen überhaupt nicht gelingen, dann ergänzt der unmittelbar gefundene Werth von $\mu$ am besten das fehlende Integral, sonst muss man durch hypothetische Annahmen über $\mu$ der Beobachtung $t_{4}$ genügen.

Wir setzen desshalb für solche Fälle voraus, dass wir für $t_{1}, t_{2}$ und $t_{3}$ die Projectionen $x_{1}, x_{2}$ und $x_{3}$ von $r$ auf eine gerade Linie kennen, deren Positionswinkel am besten derjenige ist, der den grössten Distanzen entspricht, und ferner, dass wir entweder $z$ wischen $t_{1}$ und $t_{2}$ oder $t_{2}$ und $t_{3}$ das Integral $\int \frac{\partial t}{x^{2}}$ berechnet haben, endlich sehen wir $\mu$ als bekannt an. Dann müssen wir zuerst aus:

und

$$
\begin{aligned}
t_{3}-t_{2}-x_{3} x_{2} \int_{t_{2}}^{t_{3}} \frac{c t}{x^{2}} & =\frac{\mathrm{I}}{\mu}\left[E_{3}-E_{2}-\sin \left(E_{3}-E_{2}\right)\right] \\
t_{3}-t_{1}-x_{1} x_{3}\left(\int_{t_{1}}^{t_{2}} \frac{\partial t}{x^{2}}+\int_{t_{2}}^{\frac{t_{3}}{x^{2}}} \frac{\partial t}{x^{2}}\right) & =\frac{\mathrm{I}}{\mu}\left[E_{3}-E_{1}-\sin \left(E_{3}-E_{1}\right)\right]
\end{aligned}
$$

$$
t_{2}-t_{1}-x_{1} x_{2} \int_{t_{1}}^{t_{2}} \frac{\partial t}{x_{2}}=\frac{\mathrm{I}}{\mu}\left[E_{2}-E_{1}-\sin \left(E_{2}-E_{1}\right)\right]
$$


das unbekannte Integral so durch leichte Versuche bestimmen, dass

$$
\left(E_{3}-E_{2}\right)-\left(E_{3}-E_{1}\right)+\left(E_{2}-E_{1}\right)=0
$$

Nachher stellt sich die Rechnung ganz wie oben, indem wir zuletzt, die senkrechten Abweichungen von der ge-

$$
x_{1} x_{2} \int_{t_{1}}^{t_{2}} \frac{\partial t}{x^{2}}=\frac{1}{c} r_{1} r_{2} \sin \left(c \int_{t_{1}}^{t_{2}} \frac{\partial t}{r^{2}}\right)=\frac{1}{c} r_{1} r_{2} \sin \left(R_{2}-R_{1}\right)
$$

zu rechnen; für kleine Winkel $R_{2}-R_{1}$ kann man ja den Sinus mit dem Bogen vertauschen. Das Integriren nach $\frac{1}{r^{2}}$ hat also immer die grösstmögliche Schärfe. Wenn aber die Intervalle nicht sehr klein sind, dann muss man auf diesem Wege die Constante $c$ zuerst bestimmen; dieses hat auch gar keine Schwierigkeit, wenn nur die Positionswinkel merkbar variiren. Wendet man Integrationen nach $\frac{\mathrm{I}}{\boldsymbol{r}^{2}}$ an, dann geht unsere Methode in diejenige Modification von Encke's und Savary's Methoden über, die ich in meiner Dissertation über $\gamma$ Virginis pag. 43 empfohlen habe. Mit dem durch Vergleichung der Positionswinkei mit $\int_{\frac{\partial t}{r^{2}}}^{\circ e-}$ wählten Projectionslinie mit $y$ bezéichnend, die beiden günstigsten Werthe unter $y_{1}, y_{2}$ und $y_{3}$ zur Berechnung von $a \sin A$ und $b \sin B$ nach (3) heranziehen.

Es wurde oben gesagt, dass die Berechnung der Integrale am schärfsten ist nach (10), wenn $m x+n y$ nahe ein Maximum ist. Wenn ausserdem die Integrationsintervalle klein sind, ist dieses dasselbe wie nach (7) fundenen Werthe von $c$ kennt man die linken Seiten der Gleichungen (8a) unserer Methode.

Diese ganze Classe von Methoden zeichnet sich durch die beiden Vorzige aus, erstens, dass man statt der sonst angewendeten numerischen Differentiationen, numerische Integrationen anwendet, die bekanntlich viel leichter und schärfer ausgeführt werden können; zweitens, dass man die ausgewählten geprüften und ausgeglichenen Beobachtungen strenge darstellt, welches bei den Methoden, in welchen die an sich fehlerfreien Zeiten nur zur Berechnung von $T$ und $\mu$ angewendet werden, nur zufälliger Weise gelingen kann.

Kopenhagen $188_{3}$ Jan. 20.

\section{T. N. Thiele.}

\section{Ingresso di Venere sul disco solare osservato in Roma il 6 Dicembre 1882.}

Quantunque il tempo sembrasse contrario, pure l'osservazione del primo e del secondo contatto di questo fenomeno potè farsi da noi senza veruno impedimento.

Jo osservai all' Equatoriale di Merz di 108 millimetri di apertura libera, senza diaframma, e coll' oculare polariscopio dello stesso Merz. Poteva cosi a piacimento regolare opportunamente l'intensita della luce secondo il bisogno atteso l'incertezza dello stato del cielo. L'ingrandimento adoperato pel contatto esterno fu di 50 volte, e per l'interno di 135 volte con oculare negativo.

Affine di non essere preoccupato da nessuno dei valori approssimati ottenuti dal calcolo, per notare i tempi mi servii del cronometro a tempo siderale di Frodsham, paragonato prima e dopo con un eccellente pendolo siderale di Dent.

Ecco pertanto i valori da me ottenuti ridotti in tempo medio di Roma.

Primo contatto esterno a

$$
2^{\mathrm{h}} 48^{\mathrm{m}} 27^{\mathrm{s}} \text {. } 0
$$

Primo contatto interno a

$$
3^{\text {h }} 9^{m} 3^{6:}: 2
$$

Il P. Hüninger che osservò con un cannocchiale di Sécrétan di 91 millimetri di apertura libera e con un ingrandimento di 250 volte ebbe pel

Primo contatto interno al Cronometro di Breguet

$$
3^{\text {h }} 10^{\mathrm{m}} \mathrm{8}^{\mathrm{s}}
$$

Quanto al fenomeno conosciuto sotto il nome di goutte noire nè io nè il $P$. Hüninger lo vedevamo affatto. Soltanto qualche tempo prima della comparsa del filetto lucido solare vedemmo un cerchietto rossastro attorno al pianeta dalla parte dell' orbo solare e che si chiuse prima dell' opposizione del filetto medesimo.

Questi risultati delle nostre osservazioni furono publicati nel giornale l'Osservatore Romano del giorno Io $\mathrm{Di}$ cembre $\mathrm{r} 882$ che unisco a questa communicazione.

Roma dall' osservatorio astronomico privato sul Gianicolo di $\mathbf{r}_{3}$ Gennajo I $_{88}$.

$$
\text { P. G. Stanislao Ferrari S. F., Direttore. }
$$

